and receiver operating characteristic (ROC) analysis of most common discriminating indices (Matos \& Carvalho, Mentzer Index, RDW Index, Green and King, Ehsani Index) used in differential diagnosis of these two diseases was calculated using MedCalc.v15.2 statistical software. Nonparametric nature of the CBC sample was assessed using the Kolmogorov-Smirnov test. Mann-Whitney test was used to investigate differences between the two groups. Area under the ROC curve was calculated for each index and their differences were assessed. A p-value $<0.05$ was considered significant.

Among the 5 tested indices, the Ehsani index correctly diagnosed the highest number of children with $\beta$-thalassemia, but failed to properly recognize children with IDA (sensitivity 92\%, specificity 46\%). The most commonly used Mentzer index showed similar results (sensitivity $88 \%$, specificity $48 \%$ ). The best ratio between sensitivity and specificity was observed for the new Matos \& Cavalho index (sensitivity 74\%, specificity $88 \%$ ) with highest area under the ROC curve. Pairwise comparison of ROC curves obseved a significant difference between Matos \& Cavalho index and the remaining four tested indices (RDWI $\mathrm{p}<0,0008$; Ehsani $\mathrm{p}<0,0001$; Green and King $\mathrm{p}<0,0001$; Mentzer $\mathrm{p}<0,0001)$. Kolmogorov-Smirnov test for normal distribution of $\mathrm{CBC}$ values showed a $p>0,05$ while Mann-Whitney $U$ test for independent samples showed a $p<0.05$ difference between IDA and $\beta$ - thalassemia.

Our results show that the most optimal index for discriminating between $\beta$ - thalassemia and IDA in analysed children is Matos \& Cavalho Index.

Therefore, it is more appropriate for discernment than the other analysed indexes. All indexes with low specificity (Mentzer, Ehsani, Green and King) were of low validity as they have a low proportion of IDA correctly identified as such.

\section{NON-HODGKIN LYMPHOMA IN CHILDREN: SINGLE CENTER EXPERIENCE DURING 20 YEARS}

Ernest Bilić, Zrinko Šalek*, Matej Jelić, Daniel Turudić, Petra Ivančić, Maja Pavlović, Ranka Femenić, Ana Petrović-Gluščić, Lucija Mucavac, Toni Matić, Sara Dejanović Bekić, Josip Konja, Ljubica Rajić. University Hospital Centre Zagreb

\subsection{6/archdischild-2021-europaediatrics.295}

Lymphomas are the third most common malignant disease in childhood, after leukemia and brain tumors. The aim of this study is to show stratification by gender and age as well as long term survival in pediatric patients diagnosed with NonHodgkin Lymphoma in our center.

Our retrospective analysis included 85 children with newly diagnosed NHL from January 1, 1997 to December 31, 2016. They all have been diagnosed and treated at the Department of Pediatric Hematology and oncology, University Hospital Centre Zagreb.

Out of 85 children with newly diagnosed NHL 48 of them suffered from B-cell NHL ( $\mathrm{n}=48 ; 56 \%$ ) while the rest of them had T-cell lymphoblastic lymphoma

(T-LBL) $(\mathrm{n}=20 ; 24 \%)$ or Anaplastic large-cell lymphoma (ALCL) $(\mathrm{n}=17 ; 20 \%)$. There were 25 girls and 50 boys (age $3-17$ years). Overall survival (OS) for the entire group was $78.82 \%$. Diagnose based survival is in the favor of T-LBL - $85.00 \%$ in comparison to $81.25 \%$ in B-NHL and $64.71 \%$ in ALCL.

Our survival rates are not very different from the ones in the other European countries. We expect improved survival rates after introducing novel treatment that would optimize therapeutic effect and at the same time minimize the risk of severe late toxic effects.

\section{ESTIMATION OF THE GLOMERULAR FILTRATION RATE IN CHILDREN WITH HAEMOPHILIA}

Zrinko Šalek*, Matej Šapina, Daniel Turudić, Danko Milošević, Ernest Bilić. Division of Paediatric Haematology and Oncology, Department of Paediatrics, University Hospital Centre Zagreb

10.1136/archdischild-2021-europaediatrics.296

Haemophilia is rare, inherited and severe bleeding disorder characterised with factor VIII or factor IX deficiency. The estimated glomerular filtration rate (eGFR) is one of the best-performing methods to evaluate kidney function. Glomerular filtration rate cannot be directly measured; however, it can be determined by measuring the clearance of an ideal filtration marker or estimated using predictive formulas. The aim of this preliminary study was to calculate eGFR of paediatric haemophilia patients treated in our centre and asses the correlation of eGFR calculated by creatinine-based and cystatin Cbased equations.

In our study, we included 36 boys with moderate or severe haemophilia. Out of a total of 36 patients, 27 had haemophilia A and 9 had haemophilia B.

Their mean age was $11.2 \pm 4.31$ years, with a range from 3 to 18 years. We investigated the correlation and agreement between two eGFR equations (creatinine-based 'Bedside Schwartz' equation and cystatin-C based equation). Along with applying correlation and linear regression tests, the Bland Altman test was performed to assess the agreement of the results.

Statistically significant differences were found between the mean eGFR values $(\mathrm{p}<0.001)$. No significant correlation was found between the two methods $(\mathrm{p}=0.07)$. Bland-Altman analysis results showed higher mean eGFR values of bedside Schwartz equation compared to the cystatin- $\mathrm{C}$ based formula, meaning that a significant disagreement was found between those two equations. However, within the group of haemophilia B patients, statistically significant positive correlations between the two methods were found, although still a disagreement was observed Due to the observed disagreements between eGFR within haemophilia patients, further research is needed to find the optimal measure of eGFR. We suggest extending this study on a larger cohort of patients and include other possible eGFR equations.

\section{LYMPHOMA OR ALPS?}

Cansu Koç*, Gonca Kaçar, Simge Özel Çınar, Suheyla Ocak, Nihan Burtecene, Tiraje Celkan. istanbul University-Cerrahpaşa, Cerrahpaşa Medical Faculty

10.1136/archdischild-2021-europaediatrics.297

ALPS is a rare disease characterized by chronic, non-malignant lymphoproliferation and autoimmunity. The axis of apoptosis is impaired in immunoregulation by mutation in Fas Ligand and Caspase 8 genes.

Lymphadenopathy, hepatosplenomegaly, Direct Coomb's (+), autoimmune hemolytic anemia, ITP are the most 